\title{
A LANGUAGE FOR TOPOLOGICAL STRUCTURES WHICH SATISFIES A LINDSTRÖM-THEOREM
}

BY M. ZIEGLER

\author{
Communicated January 26,1976
}

I. The language $L^{t}$. Let $L_{2}$ be the 2-sorted first order language appropriate for structures $(\mathscr{L}, \alpha, \epsilon)$, where $\mathscr{U}$ is a $L$-structure and $\alpha$ is a set of subsets of $A$. We call $(\mathcal{U}, \alpha)$ topological if $\alpha$ is a topology. We call a formula of $L_{2}$ topological if it is built up using the set quantifier $\exists X$ only in the form $\exists X(t \in$ $X \wedge \phi), X$ does not occur positively in $\phi$. ( $X$ occurs positively in $\phi$ if a free occurrence of $X$ in $\phi$ is inside the scope of an even number of negation symbols. Note. Primitive symbols are $\wedge, \neg, \exists x, \exists X$.) $L^{t}$ is defined as the set of topological sentences of $L_{2} \cdot{ }^{1}$

Lemma. (a) Define $\widetilde{\beta}=\{\bigcup s \mid s \subset \beta\}$. Then for all $\phi \in L^{t}$, ( $\left.\mathcal{U}, \beta\right) \vDash \phi$ iff $(\mathcal{Q}, \widetilde{\beta}) \vDash \phi$. (I.e. $\phi$ is invariant in the sense of Garavaglia [1] .)

(b) $\widetilde{\beta}$ is a topology iff $(\mathcal{Q}, \beta) \vDash$ top, where top is the $L^{t}$-sentence

$$
\begin{aligned}
\forall x(\exists X \wedge x \in X) \wedge \forall x \forall X(x & \in X \rightarrow \forall Y(x \in Y \\
& \rightarrow \exists Z(x \in Z \wedge \forall y(y \in Z \rightarrow y \in X \wedge y \in Y)))) .
\end{aligned}
$$

In the sequel "model" means "topological model".

COROllary (see [1]). (a) $T \subset L^{t}$ has a model iff $T \cup\{$ top $\}$ is consistent (in the 2-sorted predicate calculus of $L_{2}$ ).

(b) The set of $L^{t}$-sentences true in all models is r.e.

(c) $L^{t}$ satisfies the compactness theorem: $T$ has a model iff every finite subset of $T$ has a model.

(d) $L^{t}$ satisfies the downward Löwenheim-Skolem theorem ( $L$ countable): If $T$ has an infinite model, it has a "countable" model (थ, $\alpha$ ), i.e. A countable, $\alpha$ having a countable base.

By the methods of the next section we can prove a Lindström-

THEOREM. Let $L^{*}$ be a language for topological structures extending $L^{t}$ and satisfying the compactness theorem and the downward Löwenheim-Skolem theorem. Then $L^{*}=L^{t}$.

AMS (MOS) subject classifications (1970). Primary 02 B99, 02H99; Secondary 02G05, 54J05, 54D10.

$1_{1}$ have seen, that $L^{t}$ was first considered for topological spaces by T. A. McKee in two articles in the Z. Math. Logik Grundlagen Math. 21 (1975), 405-408 and ibid. (1976). 
COROLlary. $\phi \in L_{2}$ is invariant iff top $\vdash \phi \longleftrightarrow \psi$ for $a \psi \in L^{t}$.

So $L^{t}$ seems to be the natural language for topological structures.

REMARK. One can translate the (weaker) topological logics $L\left(Q^{n}\right), L\left(I^{n}\right)$ considered in [2], [3] into $L^{t}$. " $f$ continuous" and the separation axioms $T_{0}-$ $T_{3}$ are expressible in $L^{t}$.

II. The Ehrenfeucht-Fraissé game for $L^{t}$. There are two players, I and II, for the $\nu$-E.F. game between the two models $\mathbb{M}_{i}=\left(\mathscr{U}_{i}, \alpha_{i}\right), i \in 2$. The $k$ th move is: I chooses $i \in 2, a_{i} \in A_{i}$ and a neighbourhood $N_{i}^{k}$ of $a_{i}^{k}$. Then II chooses $a_{1-i}^{k} \in A_{1-i}$ and a neighbourhood $N_{1-i}^{k}$ of $a_{1-i}^{k}$. After $\nu$ moves II has won if $\left\{\left\langle a_{0}^{k}, a_{1}^{k}\right\rangle \mid\langle\nu\}\right.$ is a partial isomorphism between $\mathfrak{U}_{0}$ and $\mathfrak{U}_{1}$ and if for all $N_{i}^{k}$ chosen by I and all $j<\nu, a_{1-i}^{j} \in N_{1-i}^{k} \Rightarrow a_{i}^{j} \in N_{i}^{k}$.

THEOREM. (a) $\mathbb{M}_{0}$ and $\mathfrak{M}_{1}$ are $L^{t}$-elementarily equivalent iff player II has a winning strategy for all n-E.F.games between $\Re_{0}$ and $\Re_{1}, n \in \omega$.

(b) Suppose $\left|A_{i}\right| \leqslant \kappa$, and $\alpha_{i}$ possesses a base of power $\leqslant \kappa, i \in 2$. Then $\mathfrak{M}_{0} \cong M_{1}$ iff there is a winning strategy for II in the $\kappa-E . F_{.}$-game between $\mathfrak{M}_{0}$ and $\mathbb{M}_{1}$ (L finite).

REMARK. The E.F.-game described above approximates isomorphisms. One can design E.F.-games approximating other relations between models: e.g. “ $\mathbb{M}_{0} \subset \mathbb{M}_{1}$ " (i.e. $\mathscr{U}_{0}$ is a substructure of $\mathfrak{l}_{1}, \alpha_{0}$ the subspace topology), " $\mathscr{U}_{0}=$ थै ${ }_{1}, \alpha_{0}$ coarser than $\alpha_{1}$ " or " $\mathfrak{M}_{0}$ is a continuous and homomorphic image of $M_{1}$, .

III. Saturation. We call (श्), $\alpha$ ) ( $\kappa$-) saturated if there is a base $\beta$ for $\alpha$ s.t. $(\mathscr{U}, \beta)$ is $\left(\kappa_{-}\right)$saturated in the usual sense of $L_{2}$.

THEOREM. (a) Every $L^{t}$-theory has a $k$-saturated model.

(b) If $U$ is a $\kappa$-good ultrafilter on I and $|L|<\kappa$, then $\mathrm{MI}^{I} / U$ is $\kappa$-saturated $\left((\mathfrak{U}, \alpha)^{I} / U=\left(\mathscr{U}^{I} / U,\left(\widetilde{\alpha^{I}} / U\right)\right)\right)$.

(c) Two saturated, $L^{t}$-elementarily equivalent models of the same cardinality are isomorphic.

(d) Two models are $L^{t}$-elementarily equivalent iff they have isomorphic ultrapowers.

IV. Definability. $T \vdash_{t} \phi$ means: $\phi$ holds in all models of $T$.

By either the methods of II or III we can prove the

Interpolation Theorem . Assume $\vdash_{t} \phi \rightarrow \psi$ and $\phi, \psi \in L^{t}$. Then there is a $\theta \in L^{t}$ containing only nonlogical symbols which occur in both $\phi$ and $\psi$, s.t. $\vdash_{t} \phi \rightarrow \theta$ and $\vdash_{t} \theta \longrightarrow \psi$.

REMARKS. (a) The analogue of Lyndon's interpolation theorem holds.

(b) Beth's theorem follows as usual for $L^{t}$. 
Define $\phi$ to be universal if $\phi$ becomes universal in the usual sense if we erase all set-quantifiers. We have the

Preservation Theorem. Let $T \subset L^{t}, \phi, \psi \in L^{t}$. The following are equivalent: (a) For all T-models $\mathfrak{M}_{0} \supset M_{1}, M_{0} \vDash \phi \Rightarrow \Re_{1} \vDash \psi$.

(b) There is a universal $\theta \in L^{t}$ s.t. $T \vdash_{t} \phi \rightarrow \theta, T \vdash_{t} \theta \longrightarrow \psi$.

REMARK. There are syntactical characterisations of the $L^{t}$-sentences preserved by various other relations between models (e.g. the relations given in (II)).

V. Decidability. The relation " $x$ cannot be separated from $y$ by disjoint closed neighbourhoods" can be arbitrary in $T_{2}$-spaces. So we have

Theorem $(L=\varnothing)$. The theory of $T_{2}$-spaces is undecidable.

The countable $T_{3}$-spaces have a base of clopen sets. Using this and the decidability of the monadic theory of countable trees we can prove

THEOREM ( $L$ CONSISTS OF UNARY PREDICATES). The theory of $T_{3^{-}}$ spaces with a finite number of distinguished subsets is decidable.

REMARK. The $L^{t}$-elementary types can be characterized by simple invariants, e.g. all $T_{3}$-spaces without isolated points are elementarily equivalent. In fact this theory is " $\aleph_{0}$-categorical".

\section{REFERENCES}

1. S. Garavaglia, Completeness for topological languages, Notices Amer. Math. Soc. 22 (1975), p. A-473. Abstract \# 75T-E36.

2. J. A. Makowsky, An extension of topological first order logic with a back and forth characterization of elementary equivalence, 1975 (preprint).

3. J. Sgro, Completeness theorems for topological models; Completeness theorems for product topologies and continuous functions, Thesis, Yale Univ., 1974. (to appear).

TU BERLIN, FB 3, HARDENBERGSTR. 4-5, 1000 BERLIN 12, GERMANY 